\title{
Rucaparib: the past, present, and future of a newly approved PARP inhibitor for ovarian cancer
}

\author{
This article was published in the following Dove Press journal: \\ OncoTargets and Therapy \\ 19 June 2017 \\ Number of times this article has been viewed
}

\section{LE Dockery \\ CC Gunderson \\ KN Moore}

Department of Obstetrics and Gynecology, Section of Gynecologic Oncology, Stephenson Cancer Center, University of Oklahoma Health Sciences Center, Oklahoma City, OK, USA
Correspondence: KN Moore Department of Obstetrics and Gynecology, Stephenson Cancer Center, University of Oklahoma Health Sciences Center, 800 NE I0th Street, Suite 5050, Oklahoma City, OK 73I04, USA

Tel + I 40527 I 800 I x48I5I

Fax + | 405 27| 1006

Email kathleen-moore@ouhsc.edu
Abstract: Rucaparib camsylate (CO-338, AG-014699, PF-01367338) is a potent PARP-1, PARP-2, and PARP-3 inhibitor. Phase I and II studies demonstrated clinical efficacy in both $B R C A$-mutated (inclusive of germline and somatic) ovarian tumors and ovarian tumors with homologous recombination deficiency (HRD) loss of heterozygosity (LOH). Rucaparib has received the US Food and Drug Administration (FDA) approval for patients with deleterious $B R C A$ mutation (germline and/or somatic)-associated advanced ovarian cancer who have been treated with two or more chemotherapies. There is evidence to suggest that rucaparib has clinical efficacy against ovarian tumors with high HRD-LOH. Rucaparib's companion diagnostic FoundationFocus ${ }^{\mathrm{TM}} \mathrm{CDx}_{B R C A}$ test is the first FDA-approved next-generation sequencing-based companion diagnostic test designed to identify patients likely to respond to rucaparib. This article reviews the mechanisms of action, safety, approval, and indications for use of the PARP inhibitor rucaparib as well as future trials and use of rucaparib's companion diagnostic test.

Keywords: rucaparib, PARP inhibitor, ovarian cancer, companion diagnostic, loss of heterozygosity

\section{Introduction}

Epithelial ovarian cancer (including primary peritoneal and fallopian tube cancer) is the fifth leading cause of cancer-related deaths in women in the United States and the most lethal gynecologic cancer. It is estimated that in 2017, more than 22,440 women will be diagnosed with ovarian cancer leading to more than 14,080 deaths. ${ }^{1}$ Despite advances in treatment, including targeted therapies such as antiangiogenesis agents, there has been little improvement in ovarian cancer outcomes. This is in large part due to lack of effective screening mechanisms leading to predominately advanced disease at diagnosis as well as the eventual emergence of chemoresistance with recurrent disease.

Historically, recurrent ovarian cancer has been dichotomized into two categories: "platinum sensitive" and "platinum resistant." Patients with recurrent disease and a platinum-free interval (PFI; measured as time from last infusion of platinum chemotherapy to time of documented recurrence) of $\geq 6$ months are considered to be sensitive to treatment with further platinum-based chemotherapy. Those with a PFI of $<6$ months are considered "platinum resistant" with overall worse response rates and survival as compared to patients who are "platinum sensitive." 2 With advances in science including molecular profiling leading to an advanced understanding of the biology of recurrent ovarian cancer and the development of targeted therapies, the arbitrary divide between platinum-sensitive versus platinum-resistant disease is no longer clear. Targeted therapies such as antiangiogenic agents and PARP inhibitors 
have led to a more multifactorial approach to the treatment of disease recurrence. . $^{2,3}$

The PARP enzyme family (PARP-1, PARP-2, and PARP-3) is important in multiple DNA repair pathways, and defects in these repair mechanisms have been implicated in many malignancies. ${ }^{4}$ PARP binds to DNA single-strand breaks and activates the base excision repair pathway. When PARP is inhibited, single-strand breaks become double-strand breaks, which are typically repaired via homologous recombination. ${ }^{5}$ In a process known as synthetic lethality, a specific combination of vulnerabilities such as PARP inhibition and homologous recombination deficiency (HRD) leads to cell death when the single mutations alone would permit viability. ${ }^{4}$ There are currently four proposed mechanisms for PARP inhibition leading to synthetic lethality. ${ }^{6}$ In the best studied of these mechanisms, PARP inhibition specifically targets tumor cells with preexisting HRD, such as those cells possessing mutations in the $B R C A 1$ or $B R C A 2$ genes. $^{7}$ The combination of inherent low-fidelity double-strand DNA break repair and the subsequent inhibition of single- and double-strand repair pathways via PARP inhibition leads to synthetic lethality and tumor cell death. ${ }^{4}$ Additional pathways for synthetic lethality via PARP inhibition include trapping the PARP-1 enzyme on damaged DNA, effectively preventing continuation of the DNA repair process; defective BRCA1 recruitment to damaged DNA; and activation of alternative DNA repair such as error-prone nonhomologous end joining (NHEJ) or alternative end joining pathways leading to mutations or chromosomal changes and ultimately cell death. ${ }^{6}$

Ovarian cancer commonly possesses defects in DNA repair pathways such as $H R D$ due to $B R C A$ mutations or otherwise. ${ }^{8}$ Approximately $25 \%$ of new ovarian cancers harbor BRCA1/2 mutations; most of these are due to germline mutations (18\%), and approximately $7 \%$ represent somatic mutations acquired within the tumor. ${ }^{9}$ It is estimated that approximately $50 \%$ of high-grade serous ovarian carcinomas exhibit alterations in the Fanconi anemia- $B R C A$ pathway. ${ }^{10}$ Mutations in this pathway, including genes such as $R A D 51 C$, $R A D 51 D$, and BRIP1, have been associated with HRD and hereditary ovarian cancer. ${ }^{8}$ Epigenetic changes also contribute to the development of HRD. For example, silencing of $B R C A 1$ in high-grade serous ovarian cancer has been shown to occur via epigenetic changes such as BRCA1 promoter hypermethylation. ${ }^{10}$ When targeted therapy with a PARP inhibitor is combined with inherent HRD, cellular lethality results. ${ }^{11}$ This has led to extensive study of PARP inhibitors in ovarian cancer; however, whether all types of HRD are equally affected by PARP inhibition remains to be seen.
$B R C A$ mutations currently represent an important prognostic biomarker for genetic counseling and cancer risk assessment. With the development of PARP inhibition therapy, $B R C A$ testing has also become a predictive biomarker for PARP inhibitor response in ovarian cancer. ${ }^{12}$

Since the first reports of in vitro efficacy of PARP inhibitors, ${ }^{13,14}$ several different PARP inhibitors have been studied in ovarian cancer. The best studied include olaparib, veliparib, niraparib, talazoparib, and rucaparib. Each PARP inhibitor possesses subtly different mechanisms of action targeting specific PARP enzymes, including PARP-1, PARP-2, and PARP-3. ${ }^{4}$

The PARP inhibitor olaparib was the first to be approved in advanced ovarian cancer therapy for those with germline BRCA1/2 mutations. Following Phase I safety and efficacy studies, a multicenter Phase II study demonstrated response to olaparib in patients with germline $B R C A 1 / 2$ mutations and recurrent ovarian cancer, breast cancer with $\geq 3$ prior chemotherapy regimens for metastatic disease, pancreatic cancer with prior gemcitabine treatment, or prostate cancer with progression on hormonal and one systemic therapy (Study 42, ClinicalTrials.gov NCT01078662). ${ }^{15}$ A subgroup analysis of patients with germline $B R C A 1 / 2$-mutated advanced recurrent ovarian cancer and $\geq 3$ prior lines of chemotherapy revealed an overall response rate (ORR) of $34 \%$. When stratified into platinum-sensitive versus platinum-resistant disease, the efficacy was noted to be highest in platinum-sensitive (but unsuitable for further platinum therapy) disease with an ORR of $46 \%$ as compared to $30 \%$ in platinum-resistant patients. ${ }^{16}$ These findings led to the accelerated approval of olaparib in the United States in December 2014 as fourth line, and beyond single-agent therapy for the treatment of patients with recurrent ovarian cancer with germline $B R C A 1 / 2$ mutations.

\section{PARP inhibition as maintenance therapy}

Olaparib demonstrated improved progression-free survival (PFS) of 11.2 months versus 4.3 months using placebo (hazard ratio [HR] $0.18,95 \%$ CI $0.10-0.31 ; P<0.0001$ ) when used as maintenance therapy following completion of chemotherapy for platinum-sensitive recurrent ovarian cancer and has received approval for this indication in the European Union but not yet in the United States. ${ }^{12,17}$ Following recent reporting of data from SOLO2, a Phase III trial demonstrating significantly improved PFS in patients with a $B R C A 1 / 2$ mutation receiving olaparib monotherapy in the maintenance setting, the United States Food and Drug Administration (FDA) granted priority review of olaparib for 
this indication. ${ }^{18}$ In addition, the PARP inhibitor niraparib received FDA approval as maintenance therapy in women with platinum-sensitive recurrent ovarian cancer based upon the results of NOVA, a Phase III placebo-controlled trial demonstrating improved PFS in women with platinumsensitive recurrent ovarian cancer regardless of $B R C A 1 / 2$ mutation or HRD status. ${ }^{19}$

\section{HRD and PARP inhibition}

Approximately half of all high-grade serous ovarian cancers show HRD resulting in loss of or duplication of chromosomal regions and ultimately genomic loss of heterozygosity $(\mathrm{LOH}){ }^{20}$ Twenty-two percent of these are a result of a mutation in, or silencing of, other homologous recombination genes. ${ }^{21}$ Recent studies have demonstrated that even without a mutation in $B R C A$ or other known homologous recombination gene, high-grade serous ovarian carcinoma shows genomic signatures such as $\mathrm{LOH}$ indicative of downstream changes related to HRD. ${ }^{22}$ This is of particular relevance clinically, as it broadens the potential impact of PARP inhibitors in epithelial ovarian cancer not only to those with germline mutations in $B R C A 1 / 2$ but also to those with somatic $B R C A 1 / 2$ mutations, other HRD mutations, or other LOH subtypes. ${ }^{23}$ Tumor profiling to evaluate for somatic HRD mutations helps not only to identify patients who may benefit from PARP inhibition therapy but also to identify those patients who require referral to genetic counseling for further evaluation of germline mutations. Importantly, tumor profiling has demonstrated that the HRD-LOH status within a tumor may change over time as chemotherapy resistance occurs due to treatment effect. ${ }^{20}$ Somatic mutations may allow the clinician to try and identify patients who might obtain the most benefit from this class of agents if they so choose. ${ }^{24}$

There are currently many unmet needs in the treatment of ovarian cancer, particularly in the setting of recurrent disease. In particular, the optimal timing and duration of administration of PARP inhibitors has yet to be determined. Based on data obtained from patients treated with olaparib, it appears that the efficacy of PARP inhibition decreases with increasing lines of chemotherapy, ${ }^{25}$ suggesting benefit may exist for use of PARP inhibitors earlier in ovarian cancer treatment. In addition, many unanswered questions remain regarding the prediction of PARP inhibitor response in patients via the assessment of HRD or LOH. The use of the Myriad HRD assay was not predictive of response to niraparib maintenance therapy. Those receiving niraparib experienced significantly longer PFS than those receiving placebo regardless of germline $B R C A$ mutation or HRD status. This highlights the need for a better assay for the prediction of PARP inhibitor response. ${ }^{19}$ Rucaparib has recently received approval in ovarian cancer in the United States for the treatment of patients with somatic and/ or germline $B R C A$ mutation. Rucaparib is indicated for treatment one line earlier than olaparib (ie, in patients who have received two or more prior lines of chemotherapy), and unlike olaparib has approval for patients with somatic $B R C A$ mutations. In addition, the approval of rucaparib comes with a robust scientifically driven program to devise a companion biomarker to predict those patients without $B R C A$ mutations who will benefit from treatment with rucaparib.

\section{Chemistry and preclinical data Chemistry}

Rucaparib camsylate (CO-338, AG-014699, PF-01367338; 8-fluoro-2-\{4-[(methylamino)methyl]phenyl $\}-1,3,4,5$ tetrahydro-6H-azepino[5,4,3-cd]indol-6-one ((1S,4R)7,72.2.1]hept-1-yl)methanesulfonic acid salt) is a highly selective oral PARP-1, PARP-2, and PARP-3 inhibitor (Figure 1). ${ }^{26}$ The chemical formula of rucaparib camsylate is $\mathrm{C} 19 \mathrm{H} 18 \mathrm{FN} 3 \mathrm{O} \cdot \mathrm{C} 10 \mathrm{H} 16 \mathrm{O} 4 \mathrm{~S}$. Chemical profiling of PARP inhibitors revealed that while PARP inhibition is mainly targeted at PARP-1 for the treatment of cancers, this class of drugs interact with multiple PARP enzymes, interrupting DNA repair. Rucaparib, in particular, demonstrated strong stabilization of PARP-1, PARP-2, and PARP-3 and to a lesser extent PARP-4, PARP-10, PARP-12, PARP-15, and PARP-16. ${ }^{27}$ In vivo and in vitro studies of rucaparib demonstrated excellent chemosensitization and radiosensitization abilities as compared to the prior benchmark PARP inhibitor compound. In addition, mouse studies revealed no toxicity with the administration of rucaparib alone leading to the selection of rucaparib for clinical trial. ${ }^{28}$ In preclinical testing, rucaparib demonstrated antiproliferative activity in multiple cell lines carrying mutated or epigenetically silenced $B R C A 1$ or $B R C A 2$, including breast, ovarian, and pancreatic tumor cell lines. ${ }^{29}$ Further study demonstrated antitumor activity of

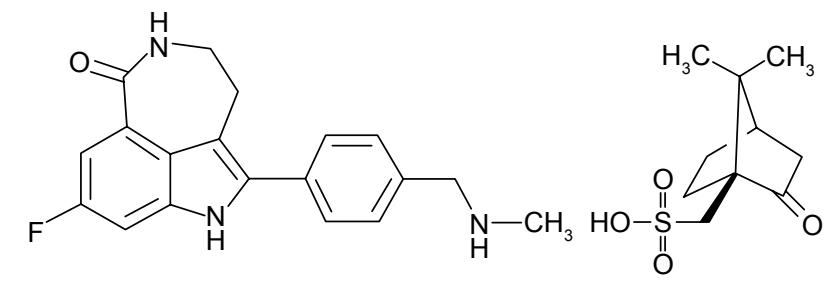

Figure I Chemical structure of rucaparib. 
rucaparib in sporadic (non- $B R C A$ mutated) human ovarian cancer cell lines both as a single agent and via potentiation of chemotherapy. ${ }^{5}$

\section{Pharmacokinetics and human metabolism}

Studies of the pharmacokinetics and human metabolism of rucaparib were performed in patients with malignancy. Table 1 includes key pharmacokinetic findings. Rucaparib may have higher solubility in the small intestine in the fed versus fasted state explaining pharmacokinetic changes in the fed state. The cytochrome P450 enzymatic pathway (primarily CYP2D6, CYP1A2, and CYP3A4) metabolizes rucaparib. Levels of CYP2D6 vary from individual to individual; however, steady-state concentrations of rucaparib have not been found to vary across CYP2D6 genotype subtypes. ${ }^{30}$

\section{Clinical trials}

Clinical safety and tolerability and pharmacokinetic and pharmacodynamic properties of rucaparib IV were first established in an open-label dose-escalation Phase I study in patients with advanced solid tumors or malignant melanoma treated with rucaparib and temozolomide. Intravenous rucaparib was administered in escalating doses to determine the PARP inhibitory dose in peripheral blood leukocytes. The PARP inhibitory dose, the dose at which the maximum achievable (at least 50\%) reduction in PARP activity occurred, was determined to be $12 \mathrm{mg} / \mathrm{m}^{2}$. There were no toxicities considered related to rucaparib alone. ${ }^{31}$

Study 10 (CO-338-010; NCT01482715), a Phase I/II open-label, multicenter trial, evaluated the maximum tolerable dose and efficacy of oral rucaparib monotherapy in patients with germline $B R C A$-mutant $\left(B R C A^{m u t}\right)$ ovarian, breast, or pancreatic tumors who had progressed on standard treatments. This study established the recommended Phase II dose of single-agent oral rucaparib as $600 \mathrm{mg}$ twice daily (b.i.d.) with an $80 \%$ ORR observed by Response Evaluation Criteria in Solid Tumors (RECIST) and cancer antigen (CA $125)$ levels. Treatment-related adverse events were predominately grades 1 and 2 , and included nausea (30\%), fatigue
(30\%), vomiting (23\%), diarrhea (13\%), and anorexia (11\%). At the recommended Phase II dose, patients reported grade $2 / 3$ anemia (29\%/29\%), thrombocytopenia (0/14\%), and neutropenia $(29 \% / 0) .{ }^{32}$ Part $2 \mathrm{~A}$ of the open-label Phase II portion of this study evaluated safety and efficacy of single-agent rucaparib in patients with recurrent, platinum-sensitive highgrade ovarian cancer possessing germline $B R C A$ mutations who had received two to four prior lines of chemotherapy. The primary end point, ORR, was assessed via RECIST criteria and revealed an ORR of $74 \%$ and a disease control rate of $77 \%$. Adverse effects (majority grade $1 / 2$ ) observed included nausea (55\%), fatigue (41\%), anemia (41\%), transient isolated aspartate aminotransferase (AST)/alanine aminotransferase (ALT) elevations (41\%), and asthenia (35\%). All the adverse events were managed via dose reduction with no discontinuation of therapy. ${ }^{33}$ Part $2 \mathrm{~B}$ of Study 10 is ongoing and will examine single-agent rucaparib in patients with relapsed high-grade ovarian cancer with germline and/ or somatic $B R C A$ mutations who have received three or more prior lines of chemotherapy.

Part 1 of a Phase II, two-part, open-label, international, multicenter study (ARIEL2 Part 1, NCT01891344) evaluated rucaparib efficacy in patients with recurrent, platinumsensitive, high-grade serous or endometrioid ovarian carcinoma who had received at least one prior platinum therapy. Enrollment of those with known $B R C A$ germline mutations was capped at 15 patients. Patients were prospectively divided into three tumor HRD subgroups: $B R C A^{\text {mut }}$ (germline or somatic), $B R C A$ wild type $\left(B R C A^{w t}\right)$ and high $\mathrm{LOH}\left(\mathrm{LOH}^{\text {high }}\right)$, or $B R C A^{w t}$ and low $\mathrm{LOH}\left(\mathrm{LOH}^{\text {low }}\right)$ utilizing the Foundation Medicine T5 next-generation sequencing assay. Tumor HRD status was $20 \% B R C A^{\text {mut }}, 40 \% \mathrm{LOH}^{\text {high }}$, $34 \% \mathrm{LOH}^{\text {low }}$, and $6 \%$ unclassified. Median PFS by HRD subgroup was 12.8 months for $B R C A^{\text {mut }}, 5.7$ months for $\mathrm{LOH}^{\text {high }}$, and 5.2 months $\mathrm{LOH}^{\text {low }}$. PFS was significantly longer in the $B R C A^{m u t}$ group (HR 0.27, 95\% CI 0.16-0.44, $P<0.0001$ ) and $\mathrm{LOH}^{\text {high }}$ group (HR 0.62, 95\% CI 0.42-0.90, $P=0.011)$ than PFS in the $\mathrm{LOH}^{\text {low }}$ group. ORR by RECIST criteria was observed more frequently in the $B R C A^{m u t}(80 \%$, $P<0.0001)$ and $\mathrm{LOH}^{\text {high }}(29 \%, P=0.0033)$ groups than

Table I Pharmacokinetics of rucaparib

\begin{tabular}{|c|c|c|c|c|c|c|c|}
\hline $\begin{array}{l}\text { Pharmacokinetic } \\
\text { parameter }\end{array}$ & Mean $T_{1 / 2}$ & Median $\mathbf{T}_{\max }$ & Clearance & Mean SS C max $_{\text {max }}$ & $\mathrm{AUC}_{0-12 \mathrm{~h}}$ & $\begin{array}{l}\text { Mean } \\
\text { bioavailability }\end{array}$ & References \\
\hline Fasted & $17-19 \mathrm{~h}$ & $1.9 \mathrm{~h}$ & I5.3-79.2 L/h & $\mathrm{I}, 940 \mathrm{ng} / \mathrm{mL}$ & $16,900 \mathrm{~h} \cdot \mathrm{ng} / \mathrm{mL}$ & $36 \%(30 \%-45 \%)$ & 28 \\
\hline After high-fat meal & & Delayed by $2.5 \mathrm{~h}$ & & Increased by $20 \%$ & $\mathrm{AUC}_{0-24 \mathrm{~h}}$ increased by $38 \%$ & & 28 \\
\hline
\end{tabular}

Notes: Mean $T_{1 / 2}$, mean terminal half-life; median $T_{\max }$, median time to maximal concentration; mean $S S C_{\max }$, mean peak serum concentration. Abbreviation: AUC, area under the curve. 
in the $\mathrm{LOH}^{\text {low }}$ group $(10 \%)$. Observed grade 3 or greater treatment-related adverse events included anemia/decreased hemoglobin (22\%), and elevated ALT or AST (12\%). Dose reductions occurred in $39 \%$ of patients, most commonly due to anemia (14\%) and nausea (11\%). ARIEL2 Part 1 demonstrated improved PFS in the $B R C A^{\text {mut }}$ and $\mathrm{LOH}^{\text {high }}$ groups treated with rucaparib as compared to the $\mathrm{LOH}^{\text {low }}$ group and provides evidence for the efficacy of tumor $\mathrm{LOH}$ assessment (particularly in tumors carrying homologous recombination gene mutations of $B R C A 1, B R C A 2$, or $R A D 51 C$ ) to predict response to rucaparib in the setting of platinum-sensitive, recurrent ovarian cancer. These results demonstrated the utility of PARP inhibition in tumors other than only those carrying a germline $B R C A 1 / 2$ mutation and served as the basis for FDA granting rucaparib breakthrough therapy designation as monotherapy treatment in recurrent ovarian cancer patients with either germline or somatic $B R C A$ mutations. ARIEL2 Part 2 will attempt to extend the findings from Part 1 by prospectively testing the $\mathrm{LOH}$ assay and the efficacy of rucaparib in patients with platinum-sensitive, platinumresistant, or platinum-refractory disease who have received three to four prior lines of chemotherapy and a treatment-free interval of greater than 6 months after primary chemotherapy. ORR has been defined as the primary outcome in Part 2, with PFS and overall survival as secondary end points. ${ }^{20}$

\section{Integrated analysis of safety}

The adverse events of 377 patients with ovarian cancer treated with rucaparib monotherapy (600 $\mathrm{mg}$ b.i.d.) in two separate open-label, single-arm trials have been combined for analysis. Dose reductions or interruptions in treatment occurred in $62 \%$ of patients, most commonly due to anemia (27\%) and fatigue/asthenia (22\%). Discontinuation of treatment occurred in $10 \%$ of patients. The median duration of treatment was 5.5 months (range $0.1-28.0$ months). Nausea and asthenia/fatigue were the most common (grades 1-4) adverse events, reported in $77 \%$ of patients. Grade $3 / 4$ nausea and asthenia/fatigue occurred in $5 \%$ and $11 \%$ of patients, respectively. Other frequently reported grade 1-4 adverse events included vomiting (46\%), anemia (44\%), and thrombocytopenia (21\%). Increase in serum creatinine (grades 1-4) was seen in $92 \%$ of patients ( $1 \%$ grade $3 / 4$ ). This is likely due to the potent inhibition of the MATE1, MATE2-K, and OCT1 transporters by rucaparib. ${ }^{34}$ Similar transporter inhibition has been reported with both olaparib and veliparib with increased serum creatinine reported with the use of olaparib. Increases in serum creatinine may be a PARP inhibitor class effect. ${ }^{35-37}$ The majority of reported creatinine elevations were grade 1 and stabilized with continued rucaparib treatment without dose modification. Grade 1-4 serum AST and ALT elevations were observed in $73 \%$ and $74 \%$ of patients, respectively (grade 3/4: 5\% AST and 13\% ALT). These elevations were asymptomatic, rarely associated with elevated bilirubin and reversed over time with continued rucaparib treatment, and no dose modification was necessary unless evidence of further hepatic impairment existed. Myelodysplastic syndrome/acute myeloid leukemia was reported in $0.5 \%$ $(2 / 377)$ of patients. ${ }^{38,39}$

On the basis of this and prior data, on December 19, 2016, the FDA granted accelerated approval for rucaparib in the thirdline treatment setting and beyond in patients with advanced ovarian cancer and germline and/or somatic $B R C A$ mutations who had been treated with two or more prior therapies. Future Phase III studies outlined in the following paragraphs are underway to evaluate rucaparib monotherapy and maintenance therapy not only in those patients with ovarian cancer and $B R C A$ mutations but also among HRD-LOH stratified groups.

ARIEL3 (NCT01968213) is an ongoing Phase III, doubleblind, randomized controlled trial evaluating rucaparib maintenance therapy in platinum-sensitive ovarian cancer (Figure 2). Eligible patients include those with high-grade serous or endometrioid epithelial ovarian, fallopian tube, or primary peritoneal cancer who have received at least two prior platinum-containing lines of chemotherapy, had a complete or partial response to the most recent platinum regimen, and are PARP naive. The primary outcome of interest is investigator-assessed PFS with overall survival, PFS by independent radiology review, and patient-reported outcomes as secondary end points. Upon enrollment, patients were stratified by gene list $\left(B R C A^{m u t}, B R C A^{w t} / \mathrm{LOH}^{\text {high }}\right.$, and $\left.B R C A^{\text {wt }} / \mathrm{LOH}^{\text {low }}\right)$, response to platinum regimen, and PFI after penultimate platinum regimen. Patients were subsequently randomized (2:1) to the treatment arm (rucaparib $600 \mathrm{mg}$ b.i.d. as maintenance) or placebo arm. ${ }^{40}$ Data from ARIEL3 are anticipated in the mid-2017.

In ARIEL4 (NCT02855944), rucaparib is being evaluated as monotherapy versus chemotherapy (monotherapy or doublet; investigator's choice) in patients with recurrent or progressive ovarian, fallopian tube, or primary peritoneal cancer with $B R C A$ germline and/or somatic mutations who have received two or more prior lines of chemotherapy. The physician's choice option allows for platinum-based chemotherapy. PFS by RECIST v1.1 is the primary outcome with overall survival and safety and tolerability of rucaparib as compared to chemotherapy as secondary outcomes $^{41}$ (Table 2). 


\section{ARIEL3: phase III study design}


Figure 2 ARIEL3 schema.

Notes: $\mathrm{N}=540$; primary end point: PFS; secondary end points: OS, health-related quality of life, and safety and tolerability; analysis will evaluate the three separate groups defined in ARIEL2. Clovis Oncology. A Study of Rucaparib as Switch Maintenance Following Platinum-Based Chemotherapy in Patients With Platinum-Sensitive, High-Grade Serous or Endometrioid Epithelial Ovarian, Primary Peritoneal or Fallopian Tube Cancer (ARIEL3). Available from https://clinicaltrials.gov/ct2/show/NCT0I9682/3?term=N CT01968213\&rank=I. NLM identifier: NCT01968213.48

Abbreviations: b.i.d., twice daily; CR, complete response; PFS, progression-free survival; p.o., by mouth; PR, partial response; OS, overall survival; R, randomized.

\section{Companion diagnostic}

In conjunction with the FDA approval of rucaparib in the setting of $B R C A$ germline and/or somatic mutations, ${ }^{20}$ the FDA also approved the FoundationFocus ${ }^{\mathrm{TM}} \mathrm{CDx}_{B R C A}$ test. This test is the first FDA-approved next-generation sequencing-based companion diagnostic test designed to identify patients likely to respond to rucaparib. Massively parallel DNA sequencing (also known as "next-generation" DNA sequencing or NGS) can be used to characterize DNA alterations including base substitutions, insertions/ deletions, copy number alterations, and fusions across cancer-associated genes in formalin-fixed and paraffin-embedded tumor specimens. ${ }^{42}$ The FoundationFocus ${ }^{\mathrm{TM}} \mathrm{CDx}_{B R C A}$ companion diagnostic test uses an NGS assay to identify patients who may benefit from rucaparib based on tumor $B R C A 1 / 2$ status. ${ }^{43}$ Validation of the HRD-LOH assay for the selection of patients beyond germline or somatic $B R C A$ status is pending the results of ARIEL3.

\section{Conclusion}

Rucaparib is an inhibitor of PARP-1, PARP-2, and PARP-3 that induces synthetic lethality in $B R C A$-mutated ovarian tumors and ovarian tumors with HRD-LOH and possesses few toxicities to normal cells. Rucaparib has received FDA approval for patients with deleterious $B R C A$ mutation (germline and/or somatic)-associated advanced ovarian cancer who have been treated with two or more chemotherapies. In addition, there is evidence to suggest that rucaparib has clinical efficacy against ovarian tumors with high HRD-LOH.
The determination of the appropriate patients to receive PARP inhibition is still ongoing. However, the responses observed following rucaparib treatment in patients with high HRD-LOH suggest that many more individuals may benefit than just those with germline or somatic $B R C A$ mutations. ${ }^{20}$ The ability of $\mathrm{LOH}$ assessment to broaden the selection of patients likely to benefit from rucaparib is still under investigation; however, it is anticipated that its further use will broaden the use of rucaparib and PARP inhibitors as a class.

The ideal timing of use of rucaparib is yet to be determined. Results from ARIEL3 and ARIEL4 will evaluate the use of rucaparib in the maintenance and treatment settings, respectively. Investigation is not yet complete regarding the benefit of rucaparib in patients with platinum-resistant disease, and ongoing studies (Study 10 Part 2B, ARIEL2 Part2, ARIEL4) are evaluating rucaparib treatment in this patient population.

Importantly, the long-term effects of PARP inhibition are not yet known, including any potential association between long-term PARP inhibitor use and myelodysplastic syndrome or acute myeloid leukemia, although observed rates of this appear to be quite low. ${ }^{19}$ In addition, the longterm use of PARP inhibitors can be associated with PARP inhibitor resistance, and the mechanisms of this are not yet fully understood. Reversion mutations leading to regained function of homologous recombination proteins (eg, $B R C A 1$, $R A D 51 C$, and $R A D 51 D$ ) in tumor cells have been shown to lead to PARP resistance. ${ }^{44,45}$ Resistance to rucaparib has also 





been demonstrated in ovarian cancer tumor cells possessing NHEJ defects. NHEJ plays a significant role in the repair of double-strand DNA breaks, is independent of HR function, and is associated with resistance to rucaparib in ex vivo primary cultures. ${ }^{46}$ Other mechanisms of resistance such as hypomorphic activity of mutant $B R C A 1$ alleles, upregulation of drug efflux pumps, and reconfiguration of the cellular DNA damage response are still under study. ${ }^{44}$

Future areas of investigation include combination therapy using PARP inhibitors such as rucaparib and other targeted therapies. Agents such as PI3K inhibitors, Wee1 kinase inhibitors, DNA topoisomerase I inhibitors, and DNA methyltransferase inhibitors are hypothesized to enhance the activity of PARP inhibitors. Preclinical data demonstrate that PARP inhibitors upregulate the PD-L1 tumorassociated immunosuppression pathway and combination therapy of PD-1 immune checkpoint blockade and PARP inhibitors significantly increase therapeutic efficacy of PARP inhibition. $^{47}$

Rucaparib possesses an acceptable safety profile, and along with its approved companion diagnostic test, represents an important new therapeutic option in the treatment of ovarian cancer. Phase III studies are currently underway to further understand the benefit-risk profile of rucaparib in the maintenance and treatment setting in advanced ovarian cancer. Furthermore, additional studies are in progress evaluating the efficacy of rucaparib in the treatment of other malignancies including breast, gastroesophageal, prostate, and other gynecologic cancers.

\section{Disclosure}

Dr CC Gunderson has served on an advisory board for Clovis Oncology. Dr KN Moore has served on advisory boards for Astra Zeneca, Clovis Oncology, Tesaro, Genentech/Roche, Advaxis, Immunogen, VBL Therapeutics, and Sutro, and also serves on the steering committees for Advaxis, Tesaro, and Genentech/Roche. Dr LE Dockery reports no conflicts of interest in this work.

\section{References}

1. American Cancer Society. Cancer Facts and Figures 2017. Atlanta: American Cancer Society; 2017.

2. Alvarez RD, Matulonis UA, Herzog TJ, Coleman RL, Monk BJ, Markman M. Moving beyond the platinum sensitive/resistant paradigm for patients with recurrent ovarian cancer. Gynecol Oncol. 2016; 141(3):405-409.

3. Pujade-Lauraine E, Combe P. Recurrent ovarian cancer. Ann Oncol. 2016;27(suppl 1):i63-i65.

4. Scott CL, Swisher EM, Kaufmann SH. Poly (ADP-ribose) polymerase inhibitors: recent advances and future development. J Clin Oncol. 2015;33(12):1397-1406.
5. Ihnen M, zu Eulenburg C, Kolarova T, et al. Therapeutic potential of the poly(ADP-ribose) polymerase inhibitor rucaparib for the treatment of sporadic human ovarian cancer. Mol Cancer Ther. 2013;12(6): 1002-1015.

6. Konecny GE, Kristeleit RS. PARP inhibitors for BRCA1/2-mutated and sporadic ovarian cancer: current practice and future directions. Br J Cancer. 2016;115(10):1157-1173.

7. Gunderson CC, Moore KN. Olaparib: an oral PARP-1 and PARP-2 inhibitor with promising activity in ovarian cancer. Future Oncol. 2015;11(5):747-757.

8. Pennington KP, Swisher EM. Hereditary ovarian cancer: beyond the usual suspects. Gynecol Oncol. 2012;124(2):347-353.

9. Pennington KP, Walsh T, Harrell MI, et al. Germline and somatic mutations in homologous recombination genes predict platinum response and survival in ovarian, fallopian tube, and peritoneal carcinomas. Clin Cancer Res. 2014;20(3):764-775.

10. Konstantinopoulos PA, Ceccaldi R, Shapiro GI, D'Andrea AD. Homologous recombination deficiency: exploiting the fundamental vulnerability of ovarian cancer. Cancer Discov. 2015;5(11):1137-1154.

11. Crafton SM, Bixel K, Hays JL. PARP inhibition and gynecologic malignancies: a review of current literature and on-going trials. Gynecol Oncol. 2016;142(3):588-596.

12. Ledermann J, Harter P, Gourley C, et al. Olaparib maintenance therapy in patients with platinum-sensitive relapsed serous ovarian cancer: a preplanned retrospective analysis of outcomes by BRCA status in a randomised phase 2 trial. Lancet Oncol. 2014;15(8):852-861.

13. Bryant HE, Schultz N, Thomas HD, et al. Specific killing of BRCA2deficient tumours with inhibitors of poly(ADP-ribose) polymerase. Nature. 2005;434(7035):913-917.

14. Farmer H, McCabe N, Lord CJ, et al. Targeting the DNA repair defect in BRCA mutant cells as a therapeutic strategy. Nature. 2005; 434(7035):917-921.

15. Kaufman B, Shapira-Frommer R, Schmutzler RK, et al. Olaparib monotherapy in patients with advanced cancer and a germline BRCA1/2 mutation. J Clin Oncol. 2015;33(3):244-250.

16. Domchek SM, Aghajanian C, Shapira-Frommer R, et al. Efficacy and safety of olaparib monotherapy in germline BRCA1/2 mutation carriers with advanced ovarian cancer and three or more lines of prior therapy. Gynecol Oncol. 2016;140(2):199-203.

17. Ledermann J, Harter P, Gourley C, et al. Olaparib maintenance therapy in platinum-sensitive relapsed ovarian cancer. $N$ Engl J Med. 2012; 366(15):1382-1392.

18. Pujade-Lauraine E, Ledermann J, Penson R, et al. Treatment with olaparib monotherapy in the maintenance setting significantly improves progression-free survival in patients with platinum-sensitive relapsed ovarian cancer: Results from the phase III SOLO2 study. Paper presented at: Society of Gynecologic Oncology Annual Meeting on Women's Cancer; 2017; National Harbor, MD.

19. Mirza MR, Monk BJ, Herrstedt J, et al. Niraparib maintenance therapy in platinum-sensitive, recurrent ovarian cancer. $N$ Engl J Med. 2016; 375(22):2154-2164.

20. Swisher EM, Lin KK, Oza AM, et al. Rucaparib in relapsed, platinumsensitive high-grade ovarian carcinoma (ARIEL2 Part 1): an international, multicentre, open-label, phase 2 trial. Lancet Oncol. 2017; 18(1):75-87.

21. Cancer Genome Atlas Research Network. Integrated genomic analyses of ovarian carcinoma. Nature. 2011;474(7353):609-615.

22. Marquard AM, Eklund AC, Joshi T, et al. Pan-cancer analysis of genomic scar signatures associated with homologous recombination deficiency suggests novel indications for existing cancer drugs. Biomark Res. 2015;3:9.

23. Moschetta M, George A, Kaye SB, Banerjee S. BRCA somatic mutations and epigenetic BRCA modifications in serous ovarian cancer. Ann Oncol. 2016;27(8):1449-1455.

24. Frey MK, Pothuri B. Homologous recombination deficiency (HRD) testing in ovarian cancer clinical practice: a review of the literature. Gynecol Oncol Res Pract. 2017;4:4. 
25. Matulonis UA, Penson RT, Domchek SM, et al. Olaparib monotherapy in patients with advanced relapsed ovarian cancer and a germline BRCA1/2 mutation: a multistudy analysis of response rates and safety. Ann Oncol. 2016;27(6):1013-1019.

26. Jenner ZB, Sood AK, Coleman RL. Evaluation of rucaparib and companion diagnostics in the PARP inhibitor landscape for recurrent ovarian cancer therapy. Future Oncol. 2016;12(12):1439-1456.

27. Wahlberg E, Karlberg T, Kouznetsova E, et al. Family-wide chemical profiling and structural analysis of PARP and tankyrase inhibitors. Nat Biotechnol. 2012;30(3):283-288.

28. Thomas HD, Calabrese CR, Batey MA, et al. Preclinical selection of a novel poly(ADP-ribose) polymerase inhibitor for clinical trial. Mol Cancer Ther. 2007;6(3):945-956.

29. Drew Y, Mulligan EA, Vong WT, et al. Therapeutic potential of poly(ADP-ribose) polymerase inhibitor AG014699 in human cancers with mutated or methylated BRCA1 or BRCA2. J Natl Cancer Inst. 2011;103(4):334-346.

30. United States Product Insert. 2017. Available from: http://www accessdata.fda.gov/drugsatfda_docs/label/2016/209115s0001bl.pdf. Accessed March 3, 2017.

31. Plummer R, Jones C, Middleton M, et al. Phase I study of the poly(ADPribose) polymerase inhibitor, AG014699, in combination with temozolomide in patients with advanced solid tumors. Clin Cancer Res. 2008; 14(23):7917-7923.

32. Kristeleit RS, Burris HA, LoRusso P, et al. Phase I/II study of oral rucaparib: final phase I results. J Clin Oncol. 2014;32(suppl 5):abstr2573.

33. Shapira-Frommer R, Oza AM, Domcheck SM, et al. A Phase II openlabel, multicenter study of single-agent rucaparib in the treatment of patients with relapsed ovarian cancer and a deleterious $B R C A$ mutation J Clin Oncol. 2015;33:abstr5513.

34. Kristeleit RS. Clinical activity of the Poly(ADP-Ribose) polymerase (PARP) inhibitor rucaparib in patients with high-grade ovarian carcinoma and a BRCA mutation: analysis of pooled data from study 10 (Parts 1, 2a, and 3) and ARIEL2 (Parts 1 and 2). Ann Oncol. 2016; 27(suppl 6):8560.

35. McCormick A, Swaisland H. In vitro assessment of the roles of drug transporters in the disposition and drug-drug interaction potential of olaparib. Xenobiotica. 1-47. Epub 2016 Oct 26.

36. Kikuchi R, Lao Y, Bow DA, et al. Prediction of clinical drug-drug interactions of veliparib (ABT-888) with human renal transporters (OAT1, OAT3, OCT2, MATE1, and MATE2K). J Pharm Sci. 2013; 102(12):4426-4432.
37. AstraZeneca Pharmaceuticals LYNPARZA (olaparib) capsules [prescribing information]. Wilmington; 2016.

38. Clovis Oncology Rubraca (rucaparib) tablets [prescribing information]. Boulder; 2016.

39. Oaknin A, Oza AV, Tinker I, et al. Integrated efficacy and safety analysis of the poly(ADP-ribose) polymerase (PARP) inhibitor rucaparib in patients (pts) with high-grade ovarian carcinoma (HGOC). Eur J Cancer. 2017;72(suppl 1):S95.

40. Swisher EM, McNeish IA, Coleman RL, et al. ARIEL 2/3: an integrated clinical trial program to assess activity of rucaparib in ovarian cancer and to identify tumor molecular characteristics predictive of response. J Clin Oncol. 2014;32(15 suppl):abstrTS5619.

41. ClinicalTrials.gov. A Study of Rucaparib Versus Chemotherapy BRCA Mutant Ovarian, Fallopian Tube, or Primary Peritoneal Cancer Patients. Available from: https:/clinicaltrials.gov/ct2/show/study/NCT0285594 4?term=ARIEL+4\&rank=1. NLM identifier: NCT02855944. Accessed January 29, 2017.

42. Frampton GM, Fichtenholtz A, Otto GA, et al. Development and validation of a clinical cancer genomic profiling test based on massively parallel DNA sequencing. Nat Biotechnol. 2013;31(11):1023-1031.

43. Lin KK, Sun L, Maloney S, et al. Quantification of genomic loss of heterozygosity enables prospective selection of ovarian cancer patients who may derive benefit from the PARP inhibitor rucaparib. Eur J Cancer. 2015;51(suppl 3):S531-S532.

44. Bouwman P, Jonkers J. Molecular pathways: how can BRCA-mutated tumors become resistant to PARP inhibitors? Clin Cancer Res. 2014 20(3):540-547.

45. Lin KK. Secondary mutations in RAD51C and RAD51D are associated with acquired resistance to the PARP inhibitor rucaparib in patients with high-grade ovarian cancer. Eur J Cancer. 2016;69(suppl 1):S13.

46. McCormick A, Donoghue P, Dixon M, et al. Ovarian cancers harbour defects in non-homologous end joining resulting in resistance to rucaparib. Clin Cancer Res. 2017;23(8):2050-2060.

47. Jiao S, Xia W, Yamaguchi H, et al. PARP inhibitor upregulates PD-L1 expression and enhances cancer-associated immunosuppression. Clin Cancer Res. Epub 2017 Feb 6.

48. Clovis Oncology. A Study of Rucaparib as Switch Maintenance Following Platinum-Based Chemotherapy in Patients With Platinum-Sensitive, High-Grade Serous or Endometrioid Epithelial Ovarian, Primary Peritoneal or Fallopian Tube Cancer (ARIEL3). Available from https:// clinicaltrials.gov/ct2/show/NCT01968213?term=NCT01968213\&rank =1. NLM identifier: NCT01968213. Accessed May 31, 2016.
OncoTargets and Therapy

\section{Publish your work in this journal}

OncoTargets and Therapy is an international, peer-reviewed, open access journal focusing on the pathological basis of all cancers, potential targets for therapy and treatment protocols employed to improve the management of cancer patients. The journal also focuses on the impact of management programs and new therapeutic agents and protocols on

\section{Dovepress}

patient perspectives such as quality of life, adherence and satisfaction. The manuscript management system is completely online and includes a very quick and fair peer-review system, which is all easy to use. Visit http://www.dovepress.com/testimonials.php to read real quotes from published authors. 